\title{
AN UNCONSTRAINED LAGRANGIAN FORMULATION AND CONSERVATION LAWS FOR THE SCHRÖDINGER MAP SYSTEM
}

\author{
PAUL SMITH
}

\begin{abstract}
We consider energy-critical Schrödinger maps from $\mathbb{R}^{2}$ into $\mathbb{S}^{2}$ and $\mathbb{H}^{2}$. Viewing such maps with respect to orthonormal frames on the pullback bundle provides a gauge field formulation of the evolution. We show that this gauge field system is the set of Euler-Lagrange equations corresponding to an action that includes a Chern-Simons term. We also introduce the stress-energy tensor and derive conservation laws. In conclusion we offer comparisons between Schrödinger maps and the closely related Chern-Simons-Schrödinger system.
\end{abstract}

\section{INTRODUCTION}

Our main purpose in this article is to derive the Schrödinger maps system at the level of the differentiated, gauged system using a variational approach. In part this is to provide a basis for resolving a certain tension: Schrödinger maps are usually introduced as constrained geometric evolution equations, whereas state-of-the-art results on Schrödinger maps are proved at the level of the gauged system, with little if any reference to the underlying map. These two formulations are related in a simple way. The gauged system is obtained by representing the differentiated Schrödinger maps system with respect to a space and time dependent orthonormal frame. Using the Frobenius theorem, one recovers the Schrödinger map system from the gauged system. In spite of this close relationship, certain gaps have persisted in what might be called the dictionary that translates between these two formulations. In particular, at the level of maps, the equation is easily seen to be Hamiltonian, though the variational formulation is not entirely satisfactory thanks to topological obstructions. At the level of the differentiated system, topological obstructions cease to exist, though different difficulties emerge, and only partial Hamiltonian and variational descriptions were known. In this article we fill in this gap, providing a natural variational formulation and Hamiltonian.

In particular we study the energy-critical Schrödinger map system with target $\mathbb{S}^{2}$ or target $\mathbb{H}^{2}$. Our first result is a natural variational formulation of the differentiated, gauged system. That is, in 92 we introduce the action. Next, in 93 , we introduce a natural stress-energy tensor and derive conservation laws. It is here that we introduce the Hamiltonian, as it may be rewritten in a simple way in terms of the stress-energy tensor. In 4 , we take up comparing Schrödinger maps with the Chern-Simons-Schrödinger system, which is suggested in part by a shared Chern-Simons term in their actions. Finally, we consider in the Appendix gradient flow and solitons from the gauged point of view. These objects are not only interesting in their own right but also are important because they are needed to construct the caloric gauge.

1.1. Geometric map equations. Suppose we have $\phi: \mathbb{R}^{d} \rightarrow M$, where $\mathbb{R}^{d}$ is Euclidean space, $M$ is a Riemannian manifold with metric $h$, and $\phi$ is a smooth map. Consider the Lagrangian

$$
\frac{1}{2} \int_{\mathbb{R}^{d}}\left\langle\partial_{j} \phi, \partial_{j} \phi\right\rangle_{h(\phi(x))} d x
$$

The author was supported by NSF grant DMS-1103877. 
where here and throughout we sum repeated Roman indices over all spatial variables. The associated Euler-Lagrange equation is

$$
\left(\phi^{*} \nabla\right)_{j} \partial_{j} \phi=0
$$

the solutions of which are called harmonic maps. Here $\nabla$ denotes the Levi-Civita connection on $M$ and $\phi^{*} \nabla$ denotes the pullback of this connection to $\mathbb{R}^{d}$. The downward gradient flow associated to (11) generates the harmonic map heat flow equation

$$
\partial_{t} \phi=\left(\phi^{*} \nabla\right)_{j} \partial_{j} \phi
$$

If the target manifold $M$ is Kähler with complex structure $J$, then to derive a Schrödinger evolution variationally we need to introduce in the action a suitable term. As this term ought only to carry one derivative, the natural pairing is with a 1-form. A drawback of this Lagrangian formulation is that there can be topological obstructions to global nonvanishing 1-forms, such as is the case with $\mathbb{S}^{2}$. This particular case may be handled by first stereographically projecting to $\mathbb{C}$ and then on that level writing down a suitable action [29, 14, though this procedure does not genuinely circumvent the fundamental topological issue. In any case, at the level of maps we are led to the Schrödinger map equation

$$
\partial_{t} \phi=J(\phi)\left(\phi^{*} \nabla\right)_{j} \partial_{j} \phi
$$

Equation (4) arises in ferromagnetism as a Heisenberg model for a ferromagnetic spin system and describes the classical spin [24, 33, 29, 6, 32].

Solutions of (3) and (4) are preserved by the rescalings

$$
\phi(t, x) \mapsto \phi\left(\lambda^{2} t, \lambda x\right) \quad \lambda>0
$$

and solutions of (2) are preserved by such scalings in the spatial variable. For each of these equations, the natural energy is given by (1), which also obeys a scaling law:

$$
E(\phi):=\frac{1}{2} \int_{\mathbb{R}^{d}}\left\langle\partial_{j} \phi, \partial_{j} \phi\right\rangle_{h(\phi(x))} d x, \quad E(\phi(x))=\lambda^{2-d} E(\phi(\lambda x))
$$

Energy is formally conserved by (4), and as noted the flow of (3) is the downward gradient flow associated to the energy. In dimension $d=2$, both the energy and the equations are preserved by rescalings, and for this reason this is called the energy-critical setting. From now on we assume $d=2$.

1.2. Gauges. One theme unifying the study of equations (2) - (4) is the use of gauges or moving frames: for each point in the domain, e.g. each $(t, x) \in I \times \mathbb{R}^{2}$ in cases (3) and (4), we choose an orthonormal basis of $T M_{\phi(t, x)}$. Frames have been used extensively in studying harmonic maps [16], and their use in the setting of Schrödinger maps in proving wellposedness was initiated in [6]. Our notation and perspective follow closely that in [37, Chapter 6]. In the energy-critical case with a surface as the target, we have one degree of freedom in our choice of orthonormal frame for each $(t, x)$. For maps from $\mathbb{R}^{2}$ into $M \in\left\{\mathbb{S}^{2}, \mathbb{H}^{2}\right\}$ evolving on some time interval $I$, a gauge choice may be represented by the diagram

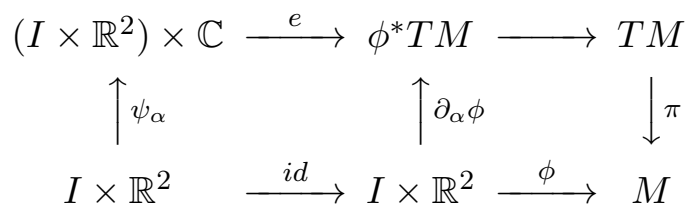

Here, for each $\alpha \in\{0,1,2\}$, the partial derivative $\partial_{\alpha} \phi$ is interpreted as a section of the pullback bundle $\phi^{*} T M$, and each $\psi_{\alpha}$ is interpreted as a section of the pullback bundle $e^{*} \phi^{*} T M$. Because the underlying manifold $M$ is complex, we complexify the tangent spaces comprising the pullback bundles. The map $e$ is identified with a choice of orthonormal frame on $\phi^{*} T M$ in the following way. For each $(t, x) \in I \times \mathbb{R}^{2}$, choose an orthonormal basis $\{v(t, x), J(\phi(t, x)) v(t, x)\}$ of $T M_{\phi(t, x)}$. Then 
let $e(t, x): \mathbb{C} \rightarrow T M_{\phi(t, x)}$ denote the linear transformation that acts according to $z \mapsto \operatorname{Re}(z) v(t, x)+$ $\operatorname{Im}(z) J(\phi(t, x)) v(t, x)$. Through $e$, the Levi-Civita connection pulls back to the covariant derivatives $D_{\alpha}:=\partial_{\alpha}+i A_{\alpha}$, which generate curvatures $F_{\alpha \beta}:=\partial_{\alpha} A_{\beta}-\partial_{\beta} A_{\alpha}$. Orthonormality of the frame ensures $A_{\alpha} \in \mathbb{R}$. The zero-torsion property of the connection enforces the compatibility condition $D_{\alpha} \psi_{\beta}=D_{\beta} \psi_{\alpha}$. Using the fact that $\mathbb{S}^{2}$ has constant curvature +1 , one may calculate directly that $F_{\alpha \beta}=\operatorname{Im}\left(\bar{\psi}_{\beta} \psi_{\alpha}\right)$. Similarly, the constant -1 curvature of $\mathbb{H}^{2}$ leads to $F_{\alpha \beta}=-\operatorname{Im}\left(\bar{\psi}_{\beta} \psi_{\alpha}\right)$. So that we can consider both cases simultaneously, we write $F_{\alpha \beta}=\mu \operatorname{Im}\left(\bar{\psi}_{\beta} \psi_{\alpha}\right)$, taking $\mu=+1$ for the sphere and $\mu=-1$ for the hyperbolic plane. Thus for any map $\phi$ and any choice of frame $e(t, x)$, it holds that

$$
F_{\alpha \beta}=\mu \operatorname{Im}\left(\bar{\psi}_{\beta} \psi_{\alpha}\right) \quad \text { and } \quad D_{\alpha} \psi_{\beta}=D_{\beta} \psi_{\alpha}
$$

These relations are all preserved by the transformations

$$
\phi \mapsto e^{-i \theta} \phi \quad A \mapsto A+d \theta
$$

where $\theta(t, x)$ is a compactly supported real-valued function (we only use time-independent functions in the case of (6) ). This gauge invariance corresponds to the freedom we have in the choice of frame $e(t, x)$.

Here and throughout we use $\partial_{0}$ and $\partial_{t}$ interchangeably. We also adopt the convention that Greek indices are allowed to assume values from the set $\{0,1,2\}$, whereas Roman indices are restricted to $\{1,2\}$, meaning that Roman indices indicate only spatial variables. Our summation conventions are that repeated Roman indices are summed over $\{1,2\}$ and that repeated Greek indices are summed over $\{0,1,2\}$.

At the gauge field level, the energy-critical harmonic maps equation (2) assumes the form

$$
\begin{cases}0 & =D_{j} \psi_{j} \\ F_{12} & =\mu \operatorname{Im}\left(\bar{\psi}_{2} \psi_{1}\right) \\ D_{1} \psi_{2} & =D_{2} \psi_{1}\end{cases}
$$

The procedure for obtaining gauge field representations of evolution equations is slightly less straightforward. For the harmonic map heat flow, for instance, we begin by pulling back the left and right hand sides of equation (3):

$$
\psi_{t}=D_{j} \psi_{j}
$$

To obtain an evolution equation from (77), we covariantly differentiate in a spatial direction by applying $D_{k}$ and then invoke the compatibility condition $D_{k} \psi_{t}=D_{t} \psi_{k}$ :

$$
D_{t} \psi_{k}=D_{k} D_{j} \psi_{j}
$$

By using the curvature relation to commute $D_{k}$ and $D_{j}$ and then applying the compatibility condition $D_{j} \psi_{k}=D_{k} \psi_{j}$, we obtain a covariant heat equation for $\psi_{k}$. All told, we arrive at the system

$$
\begin{cases}D_{t} \psi_{k} & =D_{j} D_{j} \psi_{k}-i F_{j k} \psi_{j} \\ F_{01} & =\mu \operatorname{Im}\left(\bar{\psi}_{1} D_{j} \psi_{j}\right) \\ F_{02} & =\mu \operatorname{Im}\left(\bar{\psi}_{2} D_{j} \psi_{j}\right) \\ F_{12} & =\mu \operatorname{Im}\left(\bar{\psi}_{2} \psi_{1}\right) \\ D_{1} \psi_{2} & =D_{2} \psi_{1}\end{cases}
$$

Note that we have eliminated the field $\psi_{t}$. The gauge field equations for Schrödinger maps are similarly derived. For (4), the analogue of (7) is

$$
\psi_{t}=i D_{j} \psi_{j}
$$


and we arrive at the system

$$
\begin{cases}D_{t} \psi_{k} & =i D_{j} D_{j} \psi_{k}+F_{j k} \psi_{j} \\ F_{01} & =\mu \operatorname{Re}\left(\bar{\psi}_{1} D_{j} \psi_{j}\right) \\ F_{02} & =\mu \operatorname{Re}\left(\bar{\psi}_{2} D_{j} \psi_{j}\right) \\ F_{12} & =\mu \operatorname{Im}\left(\bar{\psi}_{2} \psi_{1}\right) \\ D_{1} \psi_{2} & =D_{2} \psi_{1}\end{cases}
$$

Remark 1.1. All three of the above systems, i.e., (6), (8), and (9), are preserved by gauge transformations (5). In order to obtain well-defined flows, one must eliminate the gauge freedom by making a gauge choice. See [37, Chapter 6] for a survey of various gauge choices. It appears that the best gauge for handling arbitrary Schrödinger maps (e.g., maps without any symmetry assumption) is the caloric gauge, which was introduced in [36] in the context of wave maps and first applied to Schrödinger maps in [3]. The preferred gauge for studying Schrödinger maps with equivariant symmetry and harmonic maps is the Coulomb gauge.

Remark 1.2. It is natural to ask whether solutions of (6), (8), or (9) must arise from an underlying map. Assuming sufficient decay and regularity, this is indeed the case. We demonstrate this for Schrödinger maps into $\mathbb{S}^{2} \hookrightarrow \mathbb{R}^{3}$, where the embedding is the usual one. Let $\phi$ be a Schrödinger map and let $e_{1}, e_{2}$ denote the two vectors of the orthonormal frame $e$. Define for $\alpha=0,1,2$,

$$
\Phi=\left[\begin{array}{lll}
e_{1} & e_{2} & \phi
\end{array}\right], \quad R_{\alpha}=\left[\begin{array}{ccc}
0 & -A_{\alpha} & \operatorname{Re}\left(\psi_{\alpha}\right) \\
A_{\alpha} & 0 & \operatorname{Im}\left(\psi_{\alpha}\right) \\
-\operatorname{Re}\left(\psi_{\alpha}\right) & -\operatorname{Im}\left(\psi_{\alpha}\right) & 0
\end{array}\right]
$$

Then using (4) and the definitions, we find that $\Phi, R$ satisfy the Mayer-Lie system

$$
\partial_{\alpha} \Phi=\Phi R_{\alpha} \quad \alpha=0,1,2
$$

That (10) satisfies the Frobenius integrability condition may be described most succinctly using the Maurer-Cartan 1-form $\omega=\Phi^{-1} d \Phi$, which satisfies

$$
d \omega+\frac{1}{2}[\omega, \omega]=0
$$

In this perspective $\Phi$ is interpreted as an element of $S O(3)$ and each $R_{\alpha}$ as an element of the corresponding Lie algebra so(3).

To obtain $\Phi$ from $R$, we reverse the argument: if $(\psi, A)$ satisfy (9), then the integrability condition (11) is satisfied with $\omega=R_{\alpha} d x^{\alpha}$. If $(\psi, A)$ are rapidly decaying, then we can specify a (uniform) boundary condition for $\Phi$ at spatial infinity and recover $\Phi$ at all points by integrating in from infinity. If we have special structure such as equivariance, then instead we can specify $\Phi$ at a point $x \in \mathbb{R}^{2}$ and integrate out.

Analogous statements hold for $\mathbb{H}^{2}$ embedded in $\mathbb{R}^{3}$ endowed with the Minkowski metric. In that setting $\Phi$ is interpreted as an element of the Lorentz group $S O(2,1)$ and the $R_{\alpha}$ as elements of the associated Lie algebra $s o(2,1)$. For more details in the $\mathbb{H}^{2}$ setting and for additional related comments, see [36, §2].

\subsection{Topology.}

Definition 1.3. The charge $c_{1}$ of a vector bundle over $\mathbb{R}^{2}$ with connection $A$ is the integral

$$
c_{1}:=\frac{1}{2 \pi} \int_{\mathbb{R}^{2}} d \underline{A}=\frac{1}{2 \pi} \int_{\mathbb{R}^{2}} F_{12} d x^{1} \wedge d x^{2}
$$

The charge is also known as the first Chern number. 
The "underline" notation introduced here we will also use in the sequel: an underlined form means that we take only the spatial components of that form. For instance, if $A=A_{0} d t+A_{j} d x^{j}$, then $\underline{A}=A_{j} d x^{j}$.

Lemma 1.4. For rapidly decaying solutions of (8) or (91), charge is conserved, i.e.,

$$
\partial_{t} \frac{1}{2 \pi} \int_{\mathbb{R}^{2}} F_{12} d x=0
$$

Proof. Because $d^{2} A=0$ for any 1-form $A$,

$$
\partial_{t} F_{12}-\partial_{1} F_{02}+\partial_{2} F_{01}=0
$$

A less obvious fact is that for the system (6), charge is quantized, which is to say that it is integer-valued. At the level of maps, this follows from the Gauss-Bonnet theorem and the fact that $d \underline{A}$ is the pullback by the map of the volume form on the target. Charge in fact characterizes the homotopy class. To prove quantization at the gauge field level, one may exhaust $\mathbb{R}^{2}$ with nested discs, apply Stokes' theorem to the integral of $d \underline{A}$ over each disc, and then control the resulting integrals that arise on the boundary. The field equations are of course essential in establishing quantization. See [27, Chapter 3] for further discussion.

\section{LAGRANGIAN FORMULATION}

In this section we show that the system (9) arises as the Euler-Lagrange equations of a suitable gauge-invariant action. The difficulties encountered at the level of the map do not arise. In view of Remark 1.2, this furnishes a Lagrangian formulation for the Schrödinger map system. In carrying out variations we work formally, assuming smoothness of all quantities and assuming that fields and variations are rapidly decaying.

Theorem 2.1. The energy-critical gauged Schrödinger map system (9) is generated by the action

$$
\begin{aligned}
L_{S c h}(\psi, A):= & \int_{\mathbb{R}^{2+1}}\left[\operatorname{Re}\left(\bar{\psi}_{2} D_{t} \psi_{1}\right)-\operatorname{Im}\left(\overline{D_{j} \psi_{2}} D_{j} \psi_{1}\right)\right] d x^{1} \wedge d x^{2} \wedge d t \\
& +\frac{1}{2} \int_{\mathbb{R}^{2+1}}\left(\left|\psi_{1}\right|^{2}+\left|\psi_{2}\right|^{2}\right) d t \wedge d A+\mu \frac{1}{2} \int_{\mathbb{R}^{2+1}} A \wedge d A
\end{aligned}
$$

provided that the compatibility condition $D_{1} \psi_{2}=D_{2} \psi_{1}$ holds at the initial time.

Proof. We verify the claim by calculating the variation.

Variation of $\psi$. The variation of $\psi_{1}, \psi_{2}$ respectively give rise to the $D_{t} \psi_{2}$ and $D_{t} \psi_{1}$ evolutions of (91). Under the variation $\psi_{1} \mapsto \psi_{1}+\varepsilon \phi$, the terms linear in $\varepsilon$ from

$$
\operatorname{Re}\left(\bar{\psi}_{2} D_{t} \psi_{1}\right), \quad-\operatorname{Im}\left(\overline{D_{j} \psi_{2}} D_{j} \psi_{1}\right), \quad \frac{1}{2} F_{12}\left|\psi_{1}\right|^{2},
$$

are, respectively,

$$
\operatorname{Re}\left(\bar{\psi}_{2} D_{t} \phi\right), \quad-\operatorname{Im}\left(\overline{D_{j} \psi_{2}} D_{j} \phi\right), \quad F_{12} \operatorname{Re}\left(\bar{\psi}_{1} \phi\right)
$$

Integrating by parts in

$$
\int_{\mathbb{R}^{2+1}}\left[\operatorname{Re}\left(\bar{\psi}_{2} D_{t} \phi\right)-\operatorname{Im}\left(\overline{D_{j} \psi_{2}} D_{j} \phi\right)+F_{12} \operatorname{Re}\left(\bar{\psi}_{1} \phi\right)\right] d x d t
$$

yields

$$
\int_{\mathbb{R}^{2+1}}\left[-\operatorname{Re}\left(\bar{\phi} D_{t} \psi_{2}\right)-\operatorname{Im}\left(\bar{\phi} D_{j} D_{j} \psi_{2}\right)+F_{12} \operatorname{Re}\left(\bar{\phi} \psi_{1}\right)\right] d x d t
$$


which leads to the evolution equation

$$
D_{t} \psi_{2}=i D_{j} D_{j} \psi_{2}+F_{12} \psi_{1}
$$

Similarly, under the variation $\psi_{2} \mapsto \psi_{2}+\varepsilon \phi$ we obtain the $\varepsilon$-linear terms

$$
\operatorname{Re}\left(\bar{\phi} D_{t} \psi_{1}\right), \quad-\operatorname{Im}\left(\overline{D_{j} \phi} D_{j} \psi_{1}\right), \quad F_{12} \operatorname{Re}\left(\bar{\phi} \psi_{2}\right)
$$

which lead to the evolution equation

$$
D_{t} \psi_{1}=i D_{j} D_{j} \psi_{1}-F_{12} \psi_{2}
$$

Variation of $A$. The variation of $A_{0}$ leads to the $F_{12}$ curvature equation. Varying $A_{1}$ and $A_{2}$ respectively yield preliminary $F_{02}$ and $F_{01}$ equations. To obtain the compatibility condition $D_{1} \psi_{2}=D_{2} \psi_{1}$, we enforce it at time zero and then show using Gronwall's inequality that the condition persists. Once we have the compatibility condition, we can substitute it back into the preliminary $F_{0 j}$ equations to obtain the equations appearing in (9).

Under the variation $A \rightarrow A+\varepsilon B$, we get from $\mu \frac{1}{2} \int A \wedge d A$ the $\varepsilon$-linear term

$$
\mu B \wedge d A
$$

which can be verified using Stokes and the fact that for 1-forms $A, B$ we have $d(A \wedge B)=d A \wedge$ $B-A \wedge d B$. Upon expansion, the term appears as

$$
\mu \int_{\mathbb{R}^{2+1}}\left(B_{t} F_{12}-B_{1} F_{02}+B_{2} F_{01}\right) d x d t
$$

From $\operatorname{Re}\left(\bar{\psi}_{2} D_{t} \psi_{1}\right)$, we get from the variation of $A$ the $\varepsilon$-linear term

$$
-B_{t} \operatorname{Im}\left(\bar{\psi}_{2} \psi_{1}\right)
$$

As there are no other $A_{t}$ variation terms, we conclude from (13) and (14) that

$$
F_{12}=\mu \operatorname{Im}\left(\bar{\psi}_{2} \psi_{1}\right)
$$

We also have $\varepsilon$ terms coming from the variation of the $A_{j}$. In particular, $-\operatorname{Im}\left(\overline{D_{j} \psi_{2}} D_{j} \psi_{1}\right)$ contributes

Finally, we have to handle

$$
B_{j} \operatorname{Re}\left(\bar{\psi}_{2} D_{j} \psi_{1}\right)-B_{j} \operatorname{Re}\left(\overline{D_{j} \psi_{2}} \psi_{1}\right)
$$

$$
\frac{1}{2} \int_{\mathbb{R}^{2+1}}\left(\left|\psi_{1}\right|^{2}+\left|\psi_{2}\right|^{2}\right) d t \wedge d A
$$

To do so we first invoke Stokes to obtain

$$
\frac{1}{2} \int_{\mathbb{R}^{2+1}} d\left[\left(\left|\psi_{1}\right|^{2}+\left|\psi_{2}\right|^{2}\right) d t\right] \wedge A
$$

and then expand to get

$$
\frac{1}{2} \int_{\mathbb{R}^{2+1}}\left[A_{1} \partial_{2}\left(\left|\psi_{1}\right|^{2}+\left|\psi_{2}\right|^{2}\right)-A_{2} \partial_{1}\left(\left|\psi_{1}\right|^{2}+\left|\psi_{2}\right|^{2}\right)\right] d x^{1} \wedge d x^{2} \wedge d t
$$

Varying (17) with respect to $A$ and then expanding yields the $\varepsilon$-linear terms

$$
\int_{\mathbb{R}^{2+1}}\left[B_{1} \operatorname{Re}\left(\bar{\psi}_{1} D_{2} \psi_{1}\right)+B_{1} \operatorname{Re}\left(\bar{\psi}_{2} D_{2} \psi_{2}\right)-B_{2} \operatorname{Re}\left(\bar{\psi}_{1} D_{1} \psi_{1}\right)-B_{2} \operatorname{Re}\left(\bar{\psi}_{2} D_{1} \psi_{2}\right)\right] d x d t
$$

Comparing the $B_{1}$ terms in (13), (16), and (18) leads to

$$
\int\left[\operatorname{Re}\left(\bar{\psi}_{2} D_{1} \psi_{1}\right)-\operatorname{Re}\left(\overline{D_{1} \psi_{2}} \psi_{1}\right)+\operatorname{Re}\left(\bar{\psi}_{1} D_{2} \psi_{1}\right)+\operatorname{Re}\left(\bar{\psi}_{2} D_{2} \psi_{2}\right)-\mu F_{02}\right]=0
$$

This yields

$$
\mu F_{02}=\operatorname{Re}\left(\bar{\psi}_{2} D_{j} \psi_{j}\right)+\operatorname{Re}\left(\bar{\psi}_{1}\left(D_{2} \psi_{1}-D_{1} \psi_{2}\right)\right)
$$


Similarly, comparing $B_{2}$ terms leads to

$$
\int\left[\operatorname{Re}\left(\bar{\psi}_{2} D_{2} \psi_{1}\right)-\operatorname{Re}\left(\overline{D_{2} \psi_{2}} \psi_{1}\right)-\operatorname{Re}\left(\bar{\psi}_{1} D_{1} \psi_{1}\right)-\operatorname{Re}\left(\bar{\psi}_{2} D_{1} \psi_{2}\right)+\mu F_{01}\right]=0
$$

and hence

$$
\mu F_{01}=\operatorname{Re}\left(\bar{\psi}_{1} D_{j} \psi_{j}\right)+\operatorname{Re}\left(\bar{\psi}_{2}\left(D_{1} \psi_{2}-D_{2} \psi_{1}\right)\right)
$$

By direct calculation one may verify that (12) holds with (15), (19), and (20).

The compatibility condition. Set

$$
\Theta:=D_{1} \psi_{2}-D_{2} \psi_{1}
$$

Then

$$
D_{t} \Theta=D_{1} D_{t} \psi_{2}-D_{2} D_{t} \psi_{1}+i F_{01} \psi_{2}-i F_{02} \psi_{1}
$$

By direct calculation,

$$
D_{t} \psi_{1}=i D_{1} D_{j} \psi_{j}-i D_{2} \Theta, \quad D_{t} \psi_{2}=i D_{2} D_{j} \psi_{j}+i D_{1} \Theta
$$

which, upon substituting into (21), yield

$$
\begin{aligned}
D_{t} \Theta & =i\left(D_{1} D_{2}-D_{2} D_{1}\right) D_{j} \psi_{j}+i D_{j} D_{j} \Theta+i F_{01} \psi_{2}-i F_{02} \psi_{1} \\
& =-F_{12} D_{j} \psi_{j}+i F_{01} \psi_{2}-i F_{02} \psi_{1}+i D_{j} D_{j} \Theta
\end{aligned}
$$

Invoking (15), (19), and (20), we find

$$
-F_{12} D_{j} \psi_{j}+i F_{01} \psi_{2}-i F_{02} \psi_{1}=\mu i\left[\psi_{2} \operatorname{Re}\left(\bar{\psi}_{2} \Theta\right)+\psi_{1} \operatorname{Re}\left(\bar{\psi}_{1} \Theta\right)\right]
$$

Therefore

$$
D_{t} \Theta=i D_{j} D_{j} \Theta+\mu i\left[\psi_{2} \operatorname{Re}\left(\bar{\psi}_{2} \Theta\right)+\psi_{1} \operatorname{Re}\left(\bar{\psi}_{1} \Theta\right)\right]
$$

so that in particular

$$
\operatorname{Re}\left(\bar{\Theta} D_{t} \Theta\right)=\partial_{j} \operatorname{Re}\left(\bar{\Theta} i D_{j} \Theta\right)-\mu \operatorname{Im}\left(\bar{\Theta}\left[\psi_{2} \operatorname{Re}\left(\bar{\psi}_{2} \Theta\right)+\psi_{1} \operatorname{Re}\left(\bar{\psi}_{1} \Theta\right)\right]\right)
$$

Consequently

$$
\partial_{t} \frac{1}{2} \int_{\mathbb{R}^{2}}|\Theta|^{2} d x \leq \sup _{\mathbb{R}^{2}}\left(\left|\psi_{1}\right|^{2}+\left|\psi_{2}\right|^{2}\right) \int_{\mathbb{R}^{2}}|\Theta|^{2} d x
$$

Therefore if $\Theta=0$ at time zero, then we conclude by Gronwall's inequality that $\Theta$ is zero for all later times for which the solution exists. By time reversibility of the system, this means that the compatibility condition

$$
D_{1} \psi_{2}=D_{2} \psi_{1}
$$

holds for all times on the interval of existence provided that it holds at at least one point in the interval.

Finally, by using the compatibility condition (22) in (20) and (19), we recover the $F_{0 j}$ equations of (91).

Remark 2.2. The initial data of $(\psi, A)$ may be chosen in any way that is consistent with the curvature constraints and compatibility condition.

Remark 2.3. The time compatibility conditions $D_{0} \psi_{k}=D_{k} \psi_{0}$ are not present because we have no need for - and therefore have not introduced - the derivative field $\psi_{0}$.

Remark 2.4. A Lagrangian approach to Schrödinger maps into $\mathbb{S}^{2}$ appears in [29], though the Euler-Lagrange equations there derived do not include the compatibility condition $D_{1} \psi_{2}=D_{2} \psi_{1}$. Instead, such a constraint must be imposed. One of the key differences between our action and that introduced in [29] is that, instead of using a term quartic in $\psi$, we introduce a term that is quadratic in $\psi$ and linear in $d A$, which has the effect of coupling $\psi$ and $d A$. 


\section{Conservation laws}

Some conservation laws are written at the gauge level in [29] and derived at the level of maps in [14]. Our approach here is at the gauge level, in the spirit of [1, 7]. We begin by introducing the symmetric pseudo-stress-energy tensor $T_{\alpha \beta}$, defined by

$$
\left\{\begin{array}{l}
T_{00}=\frac{1}{2}\left(\left|\psi_{1}\right|^{2}+\left|\psi_{2}\right|^{2}\right) \\
T_{0 j}=\operatorname{Im}\left(\bar{\psi}_{\ell} D_{j} \psi_{\ell}\right) \\
T_{j k}=2 \operatorname{Re}\left(\overline{D_{j} \psi_{\ell}} D_{k} \psi_{\ell}\right)-\delta_{j k} \Delta T_{00}
\end{array}\right.
$$

Theorem 3.1. Solutions $(\psi, A)$ of the energy-critical gauged Schrödinger map system (9) satisfy the conservation law

$$
\partial_{\alpha} T_{0 \alpha}=0
$$

and the balance law

$$
\partial_{\alpha} T_{j \alpha}=2 F_{\alpha j} T_{0 \alpha}
$$

Proof. First we establish (24). Using the evolution equations in (9), we have

$$
\begin{aligned}
\frac{1}{2} \partial_{t}\left|\psi_{1}\right|^{2}=\operatorname{Re}\left(\bar{\psi}_{1} D_{t} \psi_{1}\right) & =\operatorname{Re}\left(\bar{\psi}_{1} i D_{j} D_{j} \psi_{1}\right)+\operatorname{Re}\left(\bar{\psi}_{1} F_{j 1} \psi_{j}\right) \\
& =\partial_{j} \operatorname{Re}\left(\bar{\psi}_{1} i D_{j} \psi_{1}\right)+F_{21} \operatorname{Re}\left(\bar{\psi}_{1} \psi_{2}\right)
\end{aligned}
$$

and

$$
\frac{1}{2} \partial_{t}\left|\psi_{2}\right|^{2}=\partial_{j} \operatorname{Re}\left(\bar{\psi}_{2} i D_{j} \psi_{2}\right)+F_{12} \operatorname{Re}\left(\bar{\psi}_{2} \psi_{1}\right)
$$

Consequently,

$$
\frac{1}{2} \partial_{t}\left(\left|\psi_{1}\right|^{2}+\left|\psi_{2}\right|^{2}\right)=\partial_{j} \operatorname{Re}\left(\bar{\psi}_{\ell} i D_{j} \psi_{\ell}\right)
$$

Next we show (25), which is more involved. We start by using the evolution and curvature conditions to obtain

$$
\begin{aligned}
\partial_{t} T_{0 j} & =\operatorname{Im}\left(\overline{D_{t} \psi_{\ell}} D_{j} \psi_{\ell}\right)+\operatorname{Im}\left(\bar{\psi}_{\ell} D_{t} D_{j} \psi_{\ell}\right) \\
& =\operatorname{Im}\left(\overline{i D_{k} D_{k} \psi_{\ell}} D_{j} \psi_{\ell}\right)+\operatorname{Im}\left(\overline{F_{k \ell} \psi_{k}} D_{j} \psi_{\ell}\right)+\operatorname{Im}\left(\bar{\psi}_{\ell} D_{j} D_{t} \psi_{\ell}\right)+\operatorname{Im}\left(\bar{\psi}_{\ell} i F_{0 j} \psi_{\ell}\right)
\end{aligned}
$$

The rightmost term of (26) can be rewritten as

$$
\operatorname{Im}\left(\bar{\psi}_{\ell} i F_{0 j} \psi_{\ell}\right)=F_{0 j}\left(\left|\psi_{1}\right|^{2}+\left|\psi_{2}\right|^{2}\right)=2 F_{0 j} T_{00}
$$

In view of the evolution equation, curvature conditions, and compatibility condition, the secondto-last term of (26) expands as

$$
\begin{aligned}
\operatorname{Im}\left(\bar{\psi}_{\ell} D_{j} D_{t} \psi_{\ell}\right) & =\operatorname{Im}\left(\bar{\psi}_{\ell} i D_{j} D_{k} D_{k} \psi_{\ell}\right)+\operatorname{Im}\left(\bar{\psi}_{\ell} D_{j}\left(F_{k \ell} \psi_{k}\right)\right) \\
& =\operatorname{Im}\left(\bar{\psi}_{\ell} i D_{k} D_{j} D_{k} \psi_{\ell}\right)-\operatorname{Im}\left(\bar{\psi}_{\ell} F_{j k} D_{k} \psi_{\ell}\right)+\operatorname{Im}\left(\bar{\psi}_{\ell} D_{j}\left(F_{k \ell} \psi_{k}\right)\right) \\
& =\operatorname{Im}\left(\bar{\psi}_{\ell} i D_{k} D_{j} D_{k} \psi_{\ell}\right)+\operatorname{Im}\left(\bar{\psi}_{\ell} D_{j}\left(F_{k \ell} \psi_{k}\right)\right)+F_{k j} T_{0 k} \\
& =\partial_{k} \operatorname{Im}\left(\bar{\psi}_{\ell} i D_{j} D_{k} \psi_{\ell}\right)-\operatorname{Im}\left(\bar{D}_{k} \psi_{\ell} i D_{j} D_{k} \psi_{\ell}\right)+\operatorname{Im}\left(\bar{\psi}_{\ell} D_{j}\left(F_{k \ell} \psi_{k}\right)\right)+F_{k j} T_{0 k}
\end{aligned}
$$

Appealing only to the curvature conditions and compatibility condition, we rewrite the first term of (26) as

$$
\begin{aligned}
& \operatorname{Im}\left(\overline{i D_{k} D_{k} \psi_{\ell}} D_{j} \psi_{\ell}\right)=\partial_{k} \operatorname{Im}\left(\overline{i D_{k} \psi_{\ell}} D_{j} \psi_{\ell}\right)-\operatorname{Im}\left(\overline{i D_{k} \psi_{\ell}} D_{k} D_{j} \psi_{\ell}\right) \\
&=\partial_{k} \operatorname{Im}\left(\overline{i D_{\ell} \psi_{k}} D_{j} \psi_{\ell}\right)-\operatorname{Im}\left(\overline{i D_{\ell} \psi_{k}} D_{k} D_{j} \psi_{\ell}\right) \\
& 8
\end{aligned}
$$


where we then rewrite $-\operatorname{Im}\left(\overline{i D_{\ell} \psi_{k}} D_{k} D_{j} \psi_{\ell}\right)$ as

$$
\begin{aligned}
-\operatorname{Im}\left(\overline{i D_{\ell} \psi_{k}} D_{k} D_{j} \psi_{\ell}\right) & =-\operatorname{Im}\left(\overline{i D_{\ell} \psi_{k}} D_{j} D_{k} \psi_{\ell}\right)-\operatorname{Im}\left(\overline{i D_{\ell} \psi_{k}} i F_{k j} \psi_{\ell}\right) \\
& =-\operatorname{Im}\left(\overline{i D_{\ell} \psi_{k}} D_{j} D_{k} \psi_{\ell}\right)-F_{k j} \operatorname{Im}\left(\overline{D_{k} \psi_{\ell}} \psi_{\ell}\right) \\
& =-\operatorname{Im}\left(\overline{i D_{\ell} \psi_{k}} D_{j} D_{k} \psi_{\ell}\right)+F_{k j} T_{0 k}
\end{aligned}
$$

so that

$$
\operatorname{Im}\left(\overline{i D_{k} D_{k} \psi_{\ell}} D_{j} \psi_{\ell}\right)=\partial_{k} \operatorname{Im}\left(\overline{i D_{\ell} \psi_{k}} D_{j} \psi_{\ell}\right)-\operatorname{Im}\left(\overline{i D_{\ell} \psi_{k}} D_{j} D_{k} \psi_{\ell}\right)+F_{k j} T_{0 k}
$$

Taking (27) and (28) together, we get

$$
\begin{aligned}
& \operatorname{Im}\left(\bar{\psi}_{\ell} D_{j} D_{t} \psi_{\ell}\right)+\operatorname{Im}\left(\overline{i D_{k} D_{k} \psi_{\ell}} D_{j} \psi_{\ell}\right) \\
& \quad=\partial_{k} \operatorname{Im}\left(\bar{\psi}_{\ell} i D_{j} D_{k} \psi_{\ell}\right)+\partial_{k} \operatorname{Im}\left(\overline{i D_{\ell} \psi_{k}} D_{j} \psi_{\ell}\right)+\operatorname{Im}\left(\bar{\psi}_{\ell} D_{j}\left(F_{k \ell} \psi_{k}\right)\right)+2 F_{k j} T_{0 k}
\end{aligned}
$$

Therefore

$$
\begin{aligned}
\partial_{t} T_{0 j}= & 2 F_{\alpha j} T_{0 \alpha} \\
& +\partial_{k} \operatorname{Im}\left(\bar{\psi}_{\ell} i D_{j} D_{k} \psi_{\ell}\right)+\partial_{k} \operatorname{Im}\left(\overline{i D_{\ell} \psi_{k}} D_{j} \psi_{\ell}\right)+\operatorname{Im}\left(\bar{\psi}_{\ell} D_{j}\left(F_{k \ell} \psi_{k}\right)\right)+\operatorname{Im}\left(\overline{F_{k \ell} \psi_{k}} D_{j} \psi_{\ell}\right)
\end{aligned}
$$

The last line of (29) may be rewritten as

$$
\partial_{k} \partial_{j} \operatorname{Im}\left(\bar{\psi}_{\ell} i D_{k} \psi_{\ell}\right)-2 \partial_{k} \operatorname{Im}\left(\overline{D_{j} \psi_{\ell}} i D_{k} \psi_{\ell}\right)+\partial_{j} \operatorname{Im}\left(\bar{\psi}_{\ell} F_{k \ell} \psi_{k}\right)-2 \operatorname{Im}\left(\overline{D_{j} \psi_{\ell}} F_{k \ell} \psi_{k}\right)
$$

The first term of (30) can be rewritten as $\partial_{j} \Delta T_{00}$. The third term of (30) is $\mu \partial_{j} F_{12}^{2}$. For the fourth term, we have

$$
-2 \operatorname{Im}\left(\overline{D_{j} \psi_{\ell}} F_{k \ell} \psi_{k}\right)=-2 F_{12} \mu \partial_{j} F_{12}=-\mu \partial_{j} F_{12}^{2}
$$

Therefore we may rewrite (29) as follows:

$$
\partial_{t} T_{0 j}=-2 \partial_{k} \operatorname{Im}\left(\overline{D_{j} \psi_{\ell}} i D_{k} \psi_{\ell}\right)+\partial_{j} \Delta T_{00}+2 F_{\alpha j} T_{0 \alpha}
$$

Corollary 3.2. For rapidly decaying solutions of (9), the following quantity is conserved:

$$
\frac{1}{2} \int_{\mathbb{R}^{2}}\left(\left|\psi_{1}\right|^{2}+\left|\psi_{2}\right|^{2}\right) d x
$$

Note that (31) is simply (11) written at the level of frames.

Lemma 3.3 (Hamiltonian). Let

$$
H_{S c h}:=\int_{\mathbb{R}^{2}}\left(-\operatorname{Im}\left(\overline{D_{j} \psi_{2}} D_{j} \psi_{1}\right)+\frac{1}{2}\left(\left|\psi_{1}\right|^{2}+\left|\psi_{2}\right|^{2}\right) F_{12}\right) d x^{1} \wedge d x^{2}
$$

Then, for rapidly decaying solutions of the gauged Schrödinger map system (9), it holds that

$$
H_{S c h}=\frac{1}{2} \int_{\mathbb{R}^{2}}\left(\partial_{1} T_{02}-\partial_{2} T_{01}\right) d x^{1} \wedge d x^{2}=0
$$

Proof. Using the compatibility and curvature conditions, we calculate

$$
\begin{aligned}
\operatorname{Im}\left(\overline{D_{1} \psi_{2}} D_{1} \psi_{1}\right) & =\operatorname{Im}\left(\overline{D_{2} \psi_{1}} D_{1} \psi_{1}\right) \\
& =\partial_{1} \operatorname{Im}\left(\overline{D_{2} \psi_{1}} \psi_{1}\right)-\operatorname{Im}\left(\overline{D_{1} D_{2} \psi_{1}} \psi_{1}\right) \\
& =\partial_{1} \operatorname{Im}\left(\overline{D_{2} \psi_{1}} \psi_{1}\right)-\operatorname{Im}\left(\overline{D_{2} D_{1} \psi_{1}} \psi_{1}\right)-\operatorname{Im}\left(\overline{i F_{12} \psi_{1}} \psi_{1}\right)
\end{aligned}
$$

The right hand side we may expand as

$$
\partial_{1} \operatorname{Im}\left(\overline{D_{2} \psi_{1}} \psi_{1}\right)-\partial_{2} \operatorname{Im}\left(\overline{D_{1} \psi_{1}} \psi_{1}\right)+\operatorname{Im}\left(\overline{D_{1} \psi_{1}} D_{2} \psi_{1}\right)+F_{12}\left|\psi_{1}\right|^{2}
$$

which, by virtue of the string of equalities in (33), is equal to $\operatorname{Im}\left(\overline{D_{2} \psi_{1}} D_{1} \psi_{1}\right)$. This implies

$$
2 \operatorname{Im}\left(\overline{D_{2} \psi_{1}} D_{1} \psi_{1}\right)=\partial_{1} \operatorname{Im}\left(\overline{D_{2} \psi_{1}} \psi_{1}\right)-\partial_{2} \operatorname{Im}\left(\overline{D_{1} \psi_{1}} \psi_{1}\right)+F_{12}\left|\psi_{1}\right|^{2}
$$


and hence

$$
\operatorname{Im}\left(\overline{D_{1} \psi_{2}} D_{1} \psi_{1}\right)=\frac{1}{2}\left(\partial_{1} \operatorname{Im}\left(\overline{D_{2} \psi_{1}} \psi_{1}\right)-\partial_{2} \operatorname{Im}\left(\overline{D_{1} \psi_{1}} \psi_{1}\right)+F_{12}\left|\psi_{1}\right|^{2}\right)
$$

By conjugating and reversing the roles of the indices, we similarly conclude

$$
\operatorname{Im}\left(\overline{D_{2} \psi_{2}} D_{2} \psi_{1}\right)=\frac{1}{2}\left(\partial_{2} \operatorname{Im}\left(\bar{\psi}_{2} D_{1} \psi_{2}\right)-\partial_{1} \operatorname{Im}\left(\bar{\psi}_{2} D_{2} \psi_{2}\right)+F_{12}\left|\psi_{2}\right|^{2}\right)
$$

Therefore

$$
-\operatorname{Im}\left(\overline{D_{j} \psi_{2}} D_{j} \psi_{1}\right)+\frac{1}{2}\left(\left|\psi_{1}\right|^{2}+\left|\psi_{2}\right|^{2}\right) F_{12}=\frac{1}{2}\left(\partial_{1} \operatorname{Im}\left(\bar{\psi}_{j} D_{2} \psi_{j}\right)-\partial_{2} \operatorname{Im}\left(\bar{\psi}_{j} D_{1} \psi_{j}\right)\right)
$$

Define the virial potential and Morawetz action respectively by

$$
V_{a}(t)=\int_{\mathbb{R}^{2}} a(x) T_{00} d x, \quad M_{a}(t)=\int_{\mathbb{R}^{2}} T_{0 j} \partial_{j} a d x
$$

The conservation law (24) followed by integration by parts implies

$$
\partial_{t} V_{a}(t)=M_{a}(t)
$$

We recover the generalized virial identity of [7, Lemma 3.1], adapted to the setting of Schrödinger maps.

Lemma 3.4. Let $a: \mathbb{R}^{2} \rightarrow \mathbb{R}$ and let $(\psi, A)$ be a solution of (9). Then

$$
M_{a}(T)-M_{a}(0)=\int_{0}^{T} \int_{\mathbb{R}^{2}}\left[2 \operatorname{Re}\left(\overline{D_{j} \psi_{\ell}} D_{k} \psi_{\ell}\right) \partial_{j} \partial_{k} a-\delta_{j k} T_{00} \Delta^{2} a+2 F_{\alpha j} T_{0 \alpha} \partial_{j} a\right] d x d t
$$

Proof. Using the Morawetz action, balance law, and integration by parts, we have

$$
\partial_{t} M_{a}(t)=2 \int_{\mathbb{R}^{2}}\left(T_{j k} \partial_{k} \partial_{j} a+F_{\alpha j} T_{0 \alpha} \partial_{j} a\right) d x
$$

Corollary 3.5. If a is convex, then we can further conclude that

$$
\int_{0}^{T} \int_{\mathbb{R}^{2}}\left(2 F_{\alpha j} T_{0 \alpha} \partial_{j} a-\delta_{j k} T_{00} \Delta^{2} a\right) d x d t \lesssim \sup _{[0, T]}\left|M_{a}(t)\right|
$$

Virial identities are established in the context of equivariant Schrödinger maps in [1, 2]. For virial and Morawetz identities in the context of radial Schrödinger maps, see [15].

\section{Comparison with Chern-Simons-Schrödinger}

In two spatial dimensions, the Chern-Simons-Schrödinger equation arises as the second-quantization of a nonrelativistic anyon system. For background, see [23, 9, 38, 12, 13, 20, 21, 22, 28, . Local wellposedness at high regularity is established in [5] using the Coulomb gauge and at low-regularity for small data in [26] using the heat gauge, which, in the setting of Chern-Simons-Schrödinger systems, appears to have been first introduced in [8]. Global wellposedness for large data under an equivariance ansatz and the Coulomb gauge is established at the critical regularity of $L^{2}$ in [25].

Lagrangian formulation. The action is

$$
L(\phi, A)=\frac{1}{2} \int_{\mathbb{R}^{2+1}}\left[\operatorname{Im}\left(\bar{\phi} D_{t} \phi\right)+\left|D_{x} \phi\right|^{2}-\frac{g}{2}|\phi|^{4}\right] d x^{1} \wedge d x^{2} \wedge d t+\frac{1}{2} \int_{\mathbb{R}^{2+1}} A \wedge d A
$$


with Euler-Lagrange equations

$$
\begin{cases}D_{t} \phi & =i D_{\ell} D_{\ell} \phi+i g|\phi|^{2} \phi \\ F_{01} & =-\operatorname{Im}\left(\bar{\phi} D_{2} \phi\right) \\ F_{02} & =\operatorname{Im}\left(\bar{\phi} D_{1} \phi\right) \\ F_{12} & =-\frac{1}{2}|\phi|^{2}\end{cases}
$$

both of which enjoy the gauge freedom (5). It is interesting to note that (34) is Galilean invariant, whereas (9) is not; the obstruction lies with the compatibility condition. In the above $g$ is a coupling constant. The so-called "critical coupling" is $g=1$, and this is what we consider below.

Conservation laws. For the Chern-Simons-Schrödinger system (34), we set, following [7],

$$
\begin{cases}T_{00} & =\frac{1}{2}|\phi|^{2} \\ T_{0 j} & =\operatorname{Im}\left(\bar{\phi} D_{j} \phi\right) \\ T_{j k} & =2 \operatorname{Re}\left(\overline{D_{j} \phi} D_{k} \phi\right)-\delta_{j k}\left(T_{00}+\Delta\right) T_{00}\end{cases}
$$

Here there is no distinction between the conservation law (24) and the curvature relation (12). Note that for $\phi$ not identically zero we always have

$$
\int_{\mathbb{R}^{2}} d \underline{A}=\int_{\mathbb{R}^{2}} F_{12} d x=-\int_{\mathbb{R}^{2}} T_{00} d x<0
$$

The balance law (25) is still valid in this context. Its right hand side, however, vanishes thanks to $F_{01}=-T_{02}, F_{02}=T_{01}$, and $F_{12}=-T_{00}$, so that

$$
\partial_{\alpha} T_{j \alpha}=0
$$

The conserved energy for this system is

$$
E(\phi):=\frac{1}{2} \int_{\mathbb{R}^{2}}\left(\left|D_{x} \phi\right|^{2}-\frac{1}{2}|\phi|^{4}\right) d x=\frac{1}{4} \int_{\mathbb{R}^{2}}\left(T_{11}+T_{22}\right) d x
$$

Virial identities. In spite of (35), the focusing nature of the critical coupling adds a term in the generalized virial identity with a sign that is unfavorable for establishing Morawetz estimates. In particular, this term is the $-\delta_{j k} T_{00}^{2}$ term appearing in the definition of $T_{j k}$.

Lemma 4.1. Let $a: \mathbb{R}^{2} \rightarrow \mathbb{R}$ and let $(\phi, A)$ be a solution of (34). Then the Morawetz action $M_{a}(t)$ satisfies

$$
M_{a}(T)-M_{a}(0)=\int_{0}^{T} \int_{\mathbb{R}^{2}}\left[2 \operatorname{Re}\left(\overline{D_{j} \phi} D_{k} \phi\right) \partial_{j} \partial_{k} a-\delta_{j k}\left(T_{00} \Delta^{2} a+T_{00}^{2} \Delta a\right)\right] d x d t
$$

Corollary 4.2. For $a=|x|^{2}$, it holds that

$$
\partial_{t}^{2} \int_{\mathbb{R}^{2}}|x|^{2} T_{00} d x=\partial_{t} M_{\left\{a=|x|^{2}\right\}}(t)=2 \int_{\mathbb{R}^{2}}\left(\left|D_{x} \phi\right|^{2}-2 T_{00}^{2}\right) d x=4 E(\phi)
$$

Equation (37) was used in [5] to establish the existence of finite-time blow-up solutions by taking data with negative energy or data with positive energy and sufficiently large weighted momentum. We remark that [18] constructs finite-time blow-up solutions that have charge equal to that of the ground state. The key tool in the construction is pseudo-conformal invariance. The fact that (37) holds is closely tied with exact conservation laws and pseudo-conformal invariance [37, §2.4]. In the case of Schrödinger maps, (25) is not an exact conservation law. Moreover, pseudo-conformal invariance fails to hold, the obstruction being the compatibility condition [17. If the compatibility condition were dropped, then $H_{S c h}$ introduced in (32) could be made to be nonzero, but not otherwise for maps with sufficient decay. Constructing blow-up solutions for Schrödinger maps is therefore more involved [31, 30, 34]; see also the complementary stability result [4]. 


\section{Acknowledgments}

The author thanks the anonymous referee for helpful stylistic suggestions and minor corrections.

\section{Appendix A. Gradient Flow And solitons}

Theorem A.1. The energy-critical gauged harmonic map heat flow system (8) is generated by the gradient flow of

$$
H_{H a r}(\psi, A):=\frac{1}{2} \int_{\mathbb{R}^{2}}\left(\operatorname{Re}\left(\overline{D_{j} \psi_{k}} D_{j} \psi_{k}\right)-\mu\left[\operatorname{Im}\left(\bar{\psi}_{2} \psi_{1}\right)\right]^{2}\right) d x^{1} \wedge d x^{2}-\mu \frac{1}{2} \int_{\mathbb{R}^{2}} d \underline{A} \wedge * d \underline{A}
$$

provided

$$
F_{12}=\mu \operatorname{Im}\left(\bar{\psi}_{2} \psi_{1}\right), \quad D_{1} \psi_{2}=D_{2} \psi_{1}
$$

at the initial time.

Proof. We first obtain (8) with $A_{0}=0$. A posteriori one may incorporate $A_{0} \neq 0$ in the flow and retain gauge invariance. Note that (38) itself is invariant under time-independent gauge transformations.

Varying $\psi_{1}$ leads to

$$
-\int_{\mathbb{R}^{2}}\left[\operatorname{Re}\left(\bar{\phi} D_{j} D_{j} \psi_{1}\right)+\mu \operatorname{Im}\left(\bar{\psi}_{2} \psi_{1}\right) \operatorname{Im}\left(\bar{\psi}_{2} \phi\right)\right] d x^{1} \wedge d x^{2}
$$

The associated downward gradient flow for $\psi_{1}$ is therefore

$$
\partial_{t} \psi_{1}=D_{j} D_{j} \psi_{1}+i \mu \operatorname{Im}\left(\bar{\psi}_{2} \psi_{1}\right) \psi_{2}
$$

Similarly, varying $\psi_{2}$ leads to

$$
\partial_{t} \psi_{2}=D_{j} D_{j} \psi_{2}-i \mu \operatorname{Im}\left(\bar{\psi}_{2} \psi_{1}\right) \psi_{1}
$$

Varying $A_{j}$ leads to

$$
B_{j} \operatorname{Im}\left(\bar{\psi}_{k} D_{j} \psi_{k}\right)-B_{j} \mu \partial_{k} F_{j k}
$$

Which gradient direction we choose for $A_{j}$ depends upon $\mu$ : when we couple the $F_{0 j}$ equations with (12), we choose the sign so as to obtain a forward heat evolution for $F_{12}$ rather than a backward one. Therefore we take

$$
F_{0 j}=\partial_{t} A_{j}=\mu \operatorname{Im}\left(\bar{\psi}_{k} D_{j} \psi_{k}\right)-\partial_{k} F_{j k}
$$

so that coupling (42) with (12) yields

$$
\begin{aligned}
\left(\partial_{t}-\Delta\right) F_{12} & =\mu\left[\partial_{1} \operatorname{Im}\left(\bar{\psi}_{k} D_{2} \psi_{k}\right)-\partial_{2} \operatorname{Im}\left(\bar{\psi}_{k} D_{1} \psi_{k}\right)\right] \\
& =\mu\left[-2 \operatorname{Im}\left(\overline{D_{2} \psi_{k}} D_{1} \psi_{k}\right)+\operatorname{Im}\left(\bar{\psi}_{k}\left(D_{1} D_{2}-D_{2} D_{1}\right) \psi_{k}\right)\right] \\
& =\mu\left[-2 \operatorname{Im}\left(\overline{D_{2} \psi_{k}} D_{1} \psi_{k}\right)+F_{12}\left(\left|\psi_{1}\right|^{2}+\left|\psi_{2}\right|^{2}\right)\right]
\end{aligned}
$$

On the other hand, using (40), (41), we get

$$
\begin{aligned}
\partial_{t} \operatorname{Im}\left(\bar{\psi}_{2} \psi_{1}\right) & =\operatorname{Im}\left(\bar{\psi}_{2} \partial_{t} \psi_{1}\right)-\operatorname{Im}\left(\bar{\psi}_{1} \partial_{t} \psi_{2}\right) \\
& =\operatorname{Im}\left(\bar{\psi}_{2} D_{j} D_{j} \psi_{1}\right)-\operatorname{Im}\left(\bar{\psi}_{1} D_{j} D_{j} \psi_{2}\right)+\mu \operatorname{Im}\left(\bar{\psi}_{2} \psi_{1}\right)\left(\left|\psi_{1}\right|^{2}+\left|\psi_{2}\right|^{2}\right) \\
& =\Delta \operatorname{Im}\left(\bar{\psi}_{2} \psi_{1}\right)-2 \operatorname{Im}\left(\bar{D}_{j} \psi_{2} D_{j} \psi_{1}\right)+\mu \operatorname{Im}\left(\bar{\psi}_{2} \psi_{1}\right)\left(\left|\psi_{1}\right|^{2}+\left|\psi_{2}\right|^{2}\right)
\end{aligned}
$$

so that

$$
\left(\partial_{t}-\Delta\right) \mu \operatorname{Im}\left(\bar{\psi}_{2} \psi_{1}\right)=-2 \mu \operatorname{Im}\left(\overline{D_{j} \psi_{2}} D_{j} \psi_{1}\right)+\operatorname{Im}\left(\bar{\psi}_{2} \psi_{1}\right)\left(\left|\psi_{1}\right|^{2}+\left|\psi_{2}\right|^{2}\right)
$$

Comparing (43) and (44) suggests $F_{12}=\mu \operatorname{Im}\left(\bar{\psi}_{2} \psi_{1}\right)$ and $D_{1} \psi_{2}=D_{2} \psi_{1}$, and we enforce this at the initial time. Using a Gronwall inequality argument similar to that in Theorem 2.1, we conclude that these constraints persist forward in time. In particular, set

$$
\Theta:=D_{1} \psi_{2}-D_{2} \psi_{1}, \quad \Psi:=F_{12}-\mu \operatorname{Im}\left(\bar{\psi}_{2} \psi_{1}\right)
$$


One the one hand, we can use (40), (41), and (42) to obtain

$$
\left(D_{t}-D_{j} D_{j}\right) \Theta=2 i \Psi D_{j} \psi_{j}+\mu i \psi_{j} \operatorname{Im}\left(\bar{\psi}_{j} \Theta\right)
$$

On the other hand, subtracting (44) from (43) leads to

$$
\left(\partial_{t}-\Delta\right) \Psi=2 \mu \operatorname{Im}\left(\bar{\Theta} D_{j} \psi_{j}\right)+\mu \Psi\left(\left|\psi_{1}\right|^{2}+\left|\psi_{2}\right|^{2}\right)
$$

Together (45) and (46) suffice for controlling $\int_{\mathbb{R}^{2}}\left(|\Theta|^{2}+|\Psi|^{2}\right)(t) d x$ for sufficiently regular $(\psi, A)$.

Remark A.2. One may ask whether

$$
\frac{1}{2} \int_{\mathbb{R}^{2}}\left(\operatorname{Re}\left(\overline{D_{j} \psi_{k}} D_{j} \psi_{k}\right)-\mu\left[\operatorname{Im}\left(\bar{\psi}_{2} \psi_{1}\right)\right]^{2}\right) d x^{1} \wedge d x^{2}
$$

of (38) is conserved by the Schrödinger flow (91). A straightforward calculation reveals

$$
\frac{1}{2} \partial_{t} \int_{\mathbb{R}^{2}}\left(\operatorname{Re}\left(\overline{D_{j} \psi_{k}} D_{j} \psi_{k}\right)-\mu\left[\operatorname{Im}\left(\bar{\psi}_{2} \psi_{1}\right)\right]^{2}\right) d x=\int F_{0 j} T_{0 j} d x
$$

One may rewrite the $F_{0 j} T_{0 j}$ term in various ways using

$$
\mu F_{0 j}=\partial_{k} \operatorname{Re}\left(\bar{\psi}_{j} \psi_{k}\right)-\partial_{j} T_{00}, \quad T_{0 j}=\mu \partial_{\ell} F_{j \ell}+\operatorname{Im}\left(\bar{\psi}_{j} D_{\ell} \psi_{\ell}\right)
$$

though it does not appear that this term can be expected to vanish unless $(\psi, A)$ satisfy the gauged harmonic map equations (6).

We conclude our discussion of harmonic map heat flow by noting that it is the main tool used in the construction of the caloric gauge (see [36, 35]).

We now turn to solitons, by which we mean steady states. Evident from the geometric map formulation of the evolution equations is that harmonic maps constitute the solitons for both harmonic map heat flow and Schrödinger maps.

Lemma A.3. If $(\psi, A)$ are smooth and satisfy one of the two systems

$$
\begin{cases}\left(D_{1} \pm i D_{2}\right) \psi_{1} & =0 \\ \left(D_{1} \pm i D_{2}\right) \psi_{2} & =0 \\ F_{12} & =\mu \operatorname{Im}\left(\bar{\psi}_{2} \psi_{1}\right) \\ D_{1} \psi_{2} & =D_{2} \psi_{1}\end{cases}
$$

and $F_{12} \neq 0$ at at least one point, then $(\psi, A)$ satisfy the energy-critical gauged harmonic map system (6). Moreover, if we take $A_{0}=0$, then such $(\psi, A)$ also provide stationary solutions of the gauged harmonic map heat flow system (8) and the gauged Schrödinger map system (9).

Proof. We consider solutions of (47) with " +", as solutions of (47) with " - " may be handled similarly. Invoking the compatibility condition $D_{1} \psi_{2}=D_{2} \psi_{1}$, we conclude from the first two equations of (47) that

$$
\left\{\begin{array}{l}
D_{1}\left(\psi_{1}+i \psi_{2}\right)=0 \\
D_{2}\left(\psi_{1}+i \psi_{2}\right)=0
\end{array}\right.
$$

Differentiating (48) leads to

$$
\left(D_{1} D_{2}-D_{2} D_{1}\right)\left(\psi_{1}+i \psi_{2}\right)=i F_{12}\left(\psi_{1}+i \psi_{2}\right)=0
$$

which in view of the fact that $F_{12} \neq 0$ at at least one point, implies that

$$
\psi_{1}=-i \psi_{2}
$$

holds at some $x \in \mathbb{R}^{2}$. Thanks to (48), we have for $j=1,2$ that

$$
\partial_{j}\left|\psi_{1}+i \psi_{2}\right|^{2}=0
$$


for all $x \in \mathbb{R}^{2}$. Therefore (49) also holds for all $x \in \mathbb{R}^{2}$. Together (49) and (47) imply

$$
D_{j} \psi_{j}=\left(D_{1}+i D_{2}\right) \psi_{1}=0
$$

To see that (8) and (9) are satisfied, it only remains to use the compatibility and spatial curvature conditions to write

$$
D_{j} D_{j} \psi_{k}-i F_{j k} \psi_{j}=D_{j} D_{k} \psi_{j}-i F_{j k} \psi_{j}=D_{k} D_{j} \psi_{j}=0
$$

Comparison with Chern-Simons-Schrödinger. The gradient flow of

$$
\frac{1}{2} \int_{\mathbb{R}^{2}}\left[\left|D_{x} \phi\right|^{2}-\frac{1}{2}|\phi|^{4}\right] d x^{1} \wedge d x^{2}+\frac{1}{2} \int_{\mathbb{R}^{2}} d \underline{A} \wedge * d \underline{A}
$$

yields

$$
\left\{\begin{array}{l}
D_{t} \phi=D_{\ell} D_{\ell} \phi+|\phi|^{2} \phi \\
F_{01}=-\operatorname{Im}\left(\bar{\phi} D_{1} \phi\right)-\partial_{2} F_{12} \\
F_{02}=-\operatorname{Im}\left(\bar{\phi} D_{2} \phi\right)+\partial_{1} F_{12}
\end{array}\right.
$$

which with $d^{2} A=0$ imply

$$
\left(\partial_{t}-\Delta\right) F_{12}=\partial_{1} \operatorname{Im}\left(\bar{\phi} D_{2} \phi\right)-\partial_{2} \operatorname{Im}\left(\bar{\phi} D_{1} \phi\right)
$$

In analogy with the caloric gauge for Schrödinger maps, the Chern-Simons gradient flow can be used to construct a caloric gauge for the Chern-Simons-Schrödinger system. Doing so requires initializing (50) with suitable data at time zero. Both caloric gauges can be interpreted as modifications of the Coulomb gauge. In the case of Chern-Simons-Schrödinger, the modification is not sufficient to render the caloric gauge a favorable alternative to the Coulomb gauge for the purposes of establishing wellposedness. Behind these differences in behavior is the difference between the $F_{12}$ curvature terms.

Stationary solutions of (34) are the so-called self-dual and anti-self-dual Chern-Simons solitons, which are solutions of

$$
\begin{cases}\left(D_{1} \pm i D_{2}\right) \phi & =0 \\ F_{01} & =-\operatorname{Im}\left(\bar{\phi} D_{2} \phi\right) \\ F_{02} & =\operatorname{Im}\left(\bar{\phi} D_{1} \phi\right) \\ F_{12} & =-\frac{1}{2}|\phi|^{2}\end{cases}
$$

Note that $A_{0}$ is not identically zero so long as $\phi$ is not. We refer the reader to [19, 11, 10].

\section{REFERENCES}

1. I. Bejenaru, A. Ionescu, C. E. Kenig, and D. Tataru, Equivariant Schrödinger maps in two spatial dimensions, ArXiv e-prints: 1112.6122 (2011).

2. (2012).

3. I. Bejenaru, A. D. Ionescu, C. E. Kenig, and D. Tataru, Global Schrödinger maps in dimensions $d \geq 2$ : small data in the critical Sobolev spaces, Ann. of Math. (2) 173 (2011), no. 3, 1443-1506. MR 2800718

4. I. Bejenaru and D. Tataru, Near soliton evolution for equivariant Schroedinger Maps in two spatial dimensions, ArXiv e-prints: 1009.1608 (2010).

5. L. Bergé, A. De Bouard, and J.-C. Saut, Blowing up time-dependent solutions of the planar, Chern-Simons gauged nonlinear Schrödinger equation, Nonlinearity 8 (1995), no. 2, 235-253. MR 1328596 (96b:81025)

6. Nai-Heng Chang, Jalal Shatah, and Karen Uhlenbeck, Schrödinger maps, Comm. Pure Appl. Math. 53 (2000), no. 5, 590-602. MR 1737504 (2001a:35161)

7. J. Colliander, M. Czubak, and J. Lee, Interaction Morawetz estimate for the magnetic Schrödinger equation and applications, ArXiv e-prints: 1112.4452 (2011). 
8. Sophia Demoulini, Global existence for a nonlinear Schroedinger-Chern-Simons system on a surface, Ann. Inst. H. Poincaré Anal. Non Linéaire 24 (2007), no. 2, 207-225. MR 2310693 (2008k:35439)

9. S. Deser, R. Jackiw, and S. Templeton, Topologically massive gauge theories, Ann. Physics 140 (1982), no. 2, 372-411. MR 665601 (84j:81128)

10. Gerald Dunne, R. Jackiw, So-Young Pi, and Carlo A. Trugenberger, Erratum: "Self-dual Chern-Simons solitons and two-dimensional nonlinear equations [Phys. Rev. D (3) 43 (1991), no. 4, 1332-1345; MR1104647 (92g:81120)], Phys. Rev. D (3) 45 (1992), no. 8, 3012. MR 1157129

11. Gerald V. Dunne, R. Jackiw, So-Young Pi, and Carlo A. Trugenberger, Self-dual Chern-Simons solitons and two-dimensional nonlinear equations, Phys. Rev. D (3) 43 (1991), no. 4, 1332-1345. MR 1104647 (92g:81120)

12. Z. F. Ezawa, M. Hotta, and A. Iwazaki, Breathing vortex solitons in nonrelativistic Chern-Simons gauge theory, Phys. Rev. Lett. 67 (1991), no. 4, 411-414. MR 1114940 (92f:81100)

13. _ Nonrelativistic Chern-Simons vortex solitons in external magnetic field, Phys. Rev. 44 (1991), no. D, $452-63$.

14. Manoussos G. Grillakis and Vagelis Stefanopoulos, Lagrangian formulation, energy estimates, and the Schrödinger map problem, Comm. Partial Differential Equations 27 (2002), no. 9-10, 1845-1877. MR 1941660 (2003i:58042)

15. S. Gustafson and E. Koo, Global well-posedness for $2 D$ radial Schrödinger maps into the sphere, ArXiv e-prints: 1105.5659 (2011).

16. Frédéric Hélein, Régularité des applications faiblement harmoniques entre une surface et une variété riemannienne, C. R. Acad. Sci. Paris Sér. I Math. 312 (1991), no. 8, 591-596. MR 1101039 (92e:58055)

17. Hyungjin Huh, Blow-up solutions of modified Schrödinger maps, Comm. Partial Differential Equations 33 (2008), no. 1-3, 235-243. MR 2398227 (2009i:35301)

18. _ Blow-up solutions of the Chern-Simons-Schrödinger equations, Nonlinearity 22 (2009), no. 5, 967-974. MR 2501032 (2010d:35340)

19. Hyungjin Huh and Jinmyoung Seok, The equivalence of the Chern-Simons-Schrödinger equations and its self-dual system, Journal of Mathematical Physics 54 (2013), no. 2, 021502.

20. R. Jackiw and So-Young Pi, Time-dependent Chern-Simons solitons and their quantization, Phys. Rev. D (3) 44 (1991), no. 8, 2524-2532. MR 1132645 (93d:81108)

21. __ Self-dual Chern-Simons solitons, Progr. Theoret. Phys. Suppl. (1992), no. 107, 1-40, Low-dimensional field theories and condensed matter physics (Kyoto, 1991). MR 1194691 (94d:81114)

22. R. Jackiw, So-Young Pi, and Erick J. Weinberg, Topological and nontopological solitons in relativistic and nonrelativistic Chern-Simons theory, Particles, strings and cosmology (Boston, MA, 1990), World Sci. Publ., River Edge, NJ, 1991, pp. 573-588. MR 1170608

23. R. Jackiw and S. Templeton, How super-renormalizable interactions cure their infrared divergences, Phys. Rev. 23 (1993), no. B, 2291-304.

24. L. D. Landau, Collected papers of L. D. Landau, Edited and with an introduction by D. ter Haar. Second printing, Gordon and Breach Science Publishers, New York, 1967. MR 0237287 (38 \#5577)

25. B. Liu and P. Smith, Global wellposedness of the equivariant Chern-Simons-Schrödinger equation, ArXiv e-prints: 1312.5567 (2013).

26. B. Liu, P. Smith, and D. Tataru, Local wellposedness of Chern-Simons-Schrödinger, ArXiv e-prints: 1212.1476 (2012).

27. Nicholas Manton and Paul Sutcliffe, Topological solitons, Cambridge Monographs on Mathematical Physics, Cambridge University Press, Cambridge, 2004. MR 2068924 (2006d:58020)

28. L. Martina, O. K. Pashaev, and G. Soliani, Self-dual Chern-Simons solitons in nonlinear $\sigma$-models, Modern Phys. Lett. A 8 (1993), no. 34, 3241-3250. MR 1249658 (94g:81138)

29. __ Quantization of planar ferromagnets in the Chern-Simons representation, Teoret. Mat. Fiz. 99 (1994), no. 3, 450-461. MR 1308811 (95j:81150)

30. F. Merle, P. Raphaël, and I. Rodnianski, Blow up dynamics for smooth equivariant solutions to the energy critical Schrödinger map, ArXiv e-prints: 1106.0912 (2011).

31. Frank Merle, Pierre Raphaël, and Igor Rodnianski, Blow up dynamics for smooth equivariant solutions to the energy critical Schrödinger map, C. R. Math. Acad. Sci. Paris 349 (2011), no. 5-6, 279-283. MR 2783320 (2012e:58029)

32. Andrea Nahmod, Atanas Stefanov, and Karen Uhlenbeck, On Schrödinger maps, Comm. Pure Appl. Math. 56 (2003), no. 1, 114-151. MR 1929444 (2003i:58030)

33. N. Papanicolaou and T. N. Tomaras, Dynamics of magnetic vortices, Nuclear Phys. B 360 (1991), no. 2-3, 425-462. MR 1118793 (92c:82114)

34. G. Perelman, Blow up dynamics for equivariant critical Schrödinger maps, ArXiv e-prints: 1212.6709 (2012).

35. Paul Smith, Geometric renormalization below the ground state, Int. Math. Res. Not. IMRN (2012), no. 16, 38003844. MR 2959028 
36. Terence Tao, Geometric renormalization of large energy wave maps, Journées "Équations aux Dérivées Partielles", École Polytech., Palaiseau, 2004, pp. Exp. No. XI, 32. MR 2135366 (2006i:58044)

37. , Nonlinear dispersive equations, CBMS Regional Conference Series in Mathematics, vol. 106, Published for the Conference Board of the Mathematical Sciences, Washington, DC, 2006, Local and global analysis. MR 2233925 (2008i:35211)

38. Frank Wilczek, Fractional statistics and anyon superconductivity, World Scientific Publishing Co. Inc., Teaneck, NJ, 1990. MR 1081990 (92c:81001)

University of CAlifornia, Berkeley

E-mail address: smith@math.berkeley.edu 